\title{
Disrespectful Maternity Care: Labor Room Violence in Government Health Facilities in India
}

By: Lovey Pant, Kanika Sharma, Nazar Khalid, and Nikhil Srivastav ${ }^{l}$

\begin{abstract}
The government of India endeavors to enhance the satisfaction of beneficiaries by ensuring 'Respectful Maternity Care' (RMC) at public health facilities. However, through observations in public hospitals in Madhya Pradesh and Chhattisgarh, we document widespread mistreatment of pregnant women. We found that women were subjected to different extensive verbal and physical violence. They were also humiliated for their fertility choices and had intrauterine devices (IUDs) inserted without their full knowledge and consent. A target driven approach towards contraception and population control, staff's burn-out from too many patients and long shifts, the lack of knowledge among staff on how to deal with the legitimate stress of a life and death situation, and a highly unequal society where it is socially acceptable to victimize low-ranking people, collectively contribute to different forms of violence against pregnant women in delivery rooms in India.
\end{abstract}

Keywords: labor room violence, reproductive health, contraception, quality of healthcare services, respectful maternity care, India

\footnotetext{
${ }^{1}$ The authors thank the hospital staff and pregnant women who generously allowed us to observe childbirth in delivery rooms, shared their time, and experiences with us. We are also grateful to Shilpa Bagde for helping in fieldwork, and Diane Coffey, Payal Hathi, and Aashish Gupta for helpful comments.
} 


\section{Introduction}

On a winter afternoon, Reena, a nine-months pregnant woman from rural Uttar Pradesh, started having strong labour pains. An ambulance took her to a nearby Community Health Center (CHC), but by then she had started to bleed. The CHC did not have blood transfusion facilities which Reena may have required for a complicated birth. As a result, she was referred to the government district hospital. Reena remembers, "On the way to the district hospital, I was constantly worried for my child. The thought, 'will my child survive or not?', never left my mind.”

It took Reena and her family about two hours to reach the district hospital. By then she had started to bleed profusely. While she was still in the hallway, her blood slowly dripped and stained the floor. Reena, scared and distressed, was expecting empathy from the staff. Instead, she was yelled at for dirtying the floor. She was in severe pain and started to cry. The attending doctor scolded her for crying. And when Reena couldn't control her cries, a nurse slapped her. Despite her pleading, all her companions were sent out of the Antenatal Care room she was in. Reena eventually had a stillbirth, and the disrespect she faced in the hospital exacerbated her trauma.

Like Reena, we observed numerous women facing disrespect and violence during childbirth at public health facilities in two other large north Indian states: Madya Pradesh and Chhattisgarh. As student researchers interested in newborn care and contraceptive equity, we visited two medical colleges, two district hospitals, two $\mathrm{CHCs}$, and observed thirty-one vaginal deliveries in 2018. We found widespread mistreatment of pregnant women and extensive labor room violence. We also observed that, as compared to Primary Health Centers (PHC) and CHCs, mistreatment was even more common in district hospitals and medical colleges. Physical violence and verbal abuse were not the only hardships that pregnant women had to go through. We also witnessed that women were often humiliated for their fertility choices and had intrauterine devices (IUDs) inserted without their full knowledge and consent.

These forms of labor room violence threaten women's safety, dignity, and human rights. They also have serious consequences for maternal and child health. While research is still underway, preliminary analysis reveals that such disrespect may nudge pregnant women to choose to deliver outside public hospitals: sometimes at home, and sometimes at relatively less equipped and more risky unregistered private facilities. Choosing not to deliver at public hospitals can result in worsening of child health outcomes. In several north Indian states, neonatal mortality is the 
highest among babies born in private facilities, intermediate for those born at home, and the lowest for births that happen in public facilities (Coffey et al., 2020). Therefore, improving women's experience in government health facilities is an important part of the larger health, reproductive and human rights, and human development agenda.

In this article, we begin by documenting the different forms of physical and verbal abuse that pregnant women go through in public health facilities. We move on to highlighting the pressure for family planning and insertion of IUDs. We then present a case study that brings out the complicated nature of labour room violence in India. Finally, we discuss the reasons that might trigger this problem as well as some steps that could be taken to address it. We hope that the stories presented here will provide the government and practitioners the impetus to urgently work towards making delivery rooms safe and respectful spaces free from violence.

\section{Physical violence and verbal abuse inside labor rooms}

We found delivery rooms in public hospitals to be sites of extreme stress for both staff and pregnant women. In most cases, this stress manifested into physical violence and abuse directed at the women. Of the 31 deliveries we observed, in 10 cases pregnant women were subjected to physical violence (slapped or hit), and in 17 cases they were insulted, threatened, or shouted at. To give an example, during Neerja's delivery at a medical college, she was threatened multiple times to stop her screaming. Referring to a staff member who was not on shift, a nurse told Neerja: "if she was here, she would have inserted her hand in your vagina, made her glove dirty, and then would have used the same dirty glove to slap you in the face. This is how she treats patients who scream a lot."

Physical violence inside delivery rooms can be initiated by any staff member, including the attending doctors. In fact, in several instances, we found the doctors as the primary perpetrators. A case in point is Karishma's delivery in a tertiary care hospital. Karishma was crying out in pain and this irritated the resident doctor attending the delivery. The resident hit Karishma on the leg hard and yelled: "aise kyun kar rahi hai aurat, pagal ho gayi hai kya? (Woman why are you doing this, have you gone mad?)." Sometime later, when the baby's head had just crowned in Karishma's birth canal, out of pain, she tried to slide back on the delivery table. But because it was disrupting the medical procedure, the doctor got infuriated and she hit Karishma on the leg.

Rampant physical violence in delivery rooms is often accompanied by verbal abuse and humiliation for pregnant women. In many cases, we found that women were subjected to severe 
verbal abuse when they expressed pain. Not all women could control their cries and screams because of pain, and this resulted in a cycle of yelling, scolding, humiliating, and hitting throughout the delivery.

Women were also at the receiving end of verbal abuse when accidents or unintentional disruptions to the delivery process happened. As the bedsheet accidentally fell on the floor when Sarita changed her position, the aaya yelled and slapped her saying: "if this falls again, you will be the one picking it up." These verbal abuses made pregnant women cautious of their behavior and added another burden on them. After the incident, Sarita got so cautious that she tightly clutched the bedsheet the whole time. Despite being in pain, she had two jobs to fulfill: one was to push her baby out and the second was to make sure that the bedsheet did not fall on the floor.

Similarly, another patient was subjected to insults about cleanliness when her excreta came out. To push the baby out, pregnant women must use the same muscles that we all use to push feces out, and therefore, having fecal matter on the delivery table is not an uncommon sight. Yet, the foul smell disgusted the attending doctor who humiliated the patient by saying: "Kitni budboo aa rahi hai. Pata nahi yeh log nahate bhi hain ya nahi (do these women ever take bath?)." Another staff member asked the patient insultingly: "Tum log nahate bhi ho ya nahi. Pichhle nau mahinon se nahaya bhi ya nahi? (do you people ever take bath or not? Have you taken a bath in the last nine months or not?)."

Inside the delivery rooms, such verbal abuse and humiliation often went hand in hand with threats of not finishing the necessary procedures if the patients did not obey the staff. Some of the common threats we observed went as follows: "we will leave you unstitched", "we will leave you without getting the placenta out", "you will kill your baby if you do not follow our instructions", and "if something bad happens you will be responsible for it." These frequent threats added enormous stress for the already exhausted pregnant women.

Another form of verbal abuse common in delivery rooms is the shaming of the sexuality and fertility of women. One aaya said to a patient: "You guys have fun, and then cause trouble for us. You are screaming now, but when your husband will ask you for sex, you will again run to him." Humiliating women for their fertility was observed across facilities. When Meena told the staff that she had a delivery ten months ago, everyone made fun of her for having another baby so soon. 
While there are no circumstances under which it would be appropriate for a health care provider to shame a woman who comes seeking childbirth services, these comments may particularly sting for women who do not have adequate autonomy to make decisions about when to get pregnant. As a large body of literature shows, most reproductive decisions of women in India result from spousal, familial, and societal pressures.

\section{Pressure for family planning and insertion of IUDs}

Women inside delivery rooms faced extreme pressure to undergo family planning. It is welldocumented that staff at government health facilities in India continue to get targets towards population control (Sharma 2016). Delivery rooms and sterilization camps are where these targets get materialized. Consequently, we found an aggressive push for family planning, particularly in the form of IUDs, inside the delivery rooms.

Family planning methods were pushed to the extent that ethical protocols related to contraception provision were ignored. In multiple cases, women were simply not told the likely benefits and side effects of getting an IUD. In a district hospital, Rashmi had just delivered when the IUD was inserted into her. She was not asked to give consent, and was only told that she had

already received a birth control procedure. In another incident, a nurse said to a patient after the procedure: "hum logon ne bacha na hone ke dawa dali hai. Sign kar do. Iske paise bhi mileinge (we have administered a medicine to stop future pregnancy. Sign this paper. You will also get money for this)."

Disregard for protocols and counseling was shown by the doctors too. Aarti had just delivered at a medical college when the attending doctor told her that she would be getting an IUD. Aarti refused. This annoyed the doctor who said, "I am not asking for your permission. I am telling you that you will be getting a copper-t." Aarti refused again and said that she would get sterilized. The doctor agreed with a condition: "if you want the operation, you will have to get it tomorrow." At a different medical college, a pregnant woman requested the doctor not to insert an IUD as she wanted to get a contraceptive injection. But the doctor paid no attention to her request and went ahead with an IUD insertion procedure.

In contrast to the experiences of the women described above, a handful of pregnant women who had relatively more socioeconomic advantage were not subjected to the same levels of pressure for family planning as those without it. For example, a patient whose seemingly well-off 
family was taking selfies after the delivery was not asked about getting a copper-t. Instead, the staff said among themselves: "this one is smart, she will herself use some contraceptive methods." This differential treatment suggests that social inequality also plays a role in the staff's insistence on contraception.

In instances where patients or family members resisted staff's insistence on family planning, several tactics, including misinformation and threats, were employed. A doctor told reluctant relatives of a patient: "copper-t toh lagwana hi padega, nahi toh humari job jaegi (you will have to get the copper-t, otherwise we will lose our job)." In a different incident, a nurse said: "yeh toh sarkaari niyam hai, lagana padega. Is aspataal mein delivery kara rahe ho toh yahan ka niyam manna padega (this is a government rule, you will have to get the copper-t. If you are getting delivery in this hospital, then you have to follow the rules here)." When a woman was not convinced, she was told that if she conceived again, the hospital would not provide her the childbirth services. None of this is actually true. There are no official rules that say that a woman must get an IUD if she comes to a government hospital, or that her future deliveries could not take place at a government hospital if she did not get an IUD.

Inserting an IUD right after birth without proper consent and counseling can have farreaching effects on a woman's wellbeing. If she does not understand or remember, she might be concerned about heavier periods after getting an IUD. She might also want to get pregnant again and mistakenly believe that she is unable to, which may lead to stigma and stress for her.

Extreme pressure for family planning and insertion of IUDs must be seen as another form of violence that women are subjected to inside the delivery rooms. But this form of violence is more systemic in nature. The target-driven approach to IUDs and sterilization and the push for population control in India have resulted in the sidelining of the necessary information, consent, and counseling as well as the undermining of women's contraceptive choice and autonomy.

\section{A case study in labour room violence}

We posited above that it may be relatively straightforward to stop the abuse that women face in the delivery rooms. Here we consider one particular case of violence during birth to show how there are several factors that contribute to this problem. First, we describe one birth in detail. Then, in the next section, we try to explain why the staff behaved as they did. 
Seeking assistance for childbirth, Deepa had come to a district hospital in Madhya Pradesh. After her admission, the nurse did a pelvic exam to assess the progress of her labor, took her blood pressure, and used a doppler to gauge fetal distress by hearing its heartbeat. The fetus was doing well, and Deepa was in the second stage of labour. This meant that it was time for Deepa to push the baby out. Her contractions were extremely intense and, because she had already been in labor for some time, she was also feeling tired.

The attending nurse instructed Deepa to lie down in the lithotomy position ${ }^{\mathrm{i}}$, but she was having trouble in keeping her legs in the stirrups. With every following contraction, she was feeling more exhausted. Seeing Deepa struggle, the attending nurse remarked: "aaj to sab mote-mote [mahilaaein] hi aa rahe hain (all the fat women are coming today)." The ward aaya followed, "haan isse pehle jo aaaye thein, wo bhi mutallo hi thi (yes, one who came before her was also fat)." While it is uncomfortable for any laboring woman to keep her legs up in stirrups, perhaps it is particularly difficult for women on the higher side of the Body Mass Index (BMI) distribution. Ignoring this, the attending staff did not provide support or suggest a more comfortable position, and instead ridiculed and shamed Deepa for her weight.

When Deepa could not keep her legs in the stirrups, the staff told her to keep lying on the delivery table, hold her ankles instead, and then try pushing the baby out. But Deepa was still unable to follow nurse's instructions. This new advice did not work either; and anticipating that Deepa's delivery would still take some time, the staff nurse checked on the fetus's heartbeat and left Deepa to attend another patient who had just arrived. All this while, when she felt contractions, Deepa continued to push.

The nurse came back to Deepa in a while. This time, the nurse looked a little more composed and determined. She stepped on a stool that was lying next to the delivery table and started to apply pressure on Deepa's abdomen. It went for several minutes until when the aaya, who was now performing a pelvic exam, announced: "delivery mein abhi thoda samay hai. Bachche dani ka rasta khula toh hai par woh abhi bhi thoda sakra hai (there is still some time in the delivery. The cervix has dilated; however, it is not wide enough for the baby to crown)." The time for a change of shift was close, but Deepa was nowhere close to being done.

The new nurse started her shift by checking the fetus's heartbeat and found it normal. Deepa was told to push but once again she failed to fulfill the nurse's expectations. Despite 
knowing how long Deepa had been on the delivery table, the nurse got irritated. She performed another pelvic exam and concluded, "bachcha bas aane he wala hai (baby is just about to arrive)." To inform her next steps, she reached out for a doppler and once again checked the fetus's heartbeat. But this time a clear beat was not audible, a sign that the fetus might be in distress. Worry flashed across the nurse's face. She tried to change her position to listen to the heartbeat again, but Deepa's leg was coming in her way. This made the nurse angry and she hit and pushed Deepa's leg aside.

It was not clear if the nurse was able to hear the fetus's heartbeat, but her actions revealed that she had decided that it was time for the baby to be born. By now, the aaya was already standing on the stool and the cycle of pushing, putting pressure on the abdomen, and yelling started anew. But none of it was working. This made the aaya-who had her dirty slippers on -leap over to the delivery table. With her feet firmly planted at the edge of the delivery table, the aaya stood on top of Deepa. Once in balance, she started exerting even more pressure on Deepa's stomach. In exhaustion and rage, she even slapped Deepa several times. A female family member of Deepa's and her village's Accredited Social Health Activist (ASHA) saw this, but instead of intervening, they joined in: "haan haan, peeto peeto! (yes, yes, slap her!)."

After much struggle, the baby crowned: its head started to show in Deepa's birth canal. Now with every push it was sliding down. But suddenly the baby stopped making progress, it had gotten stuck. This time because the baby's umbilical cord had looped around its neck. The attending nurse then swiftly grabbed a pair of scissors to cut the umbilical cord, pulled the baby out, cleaned it, and passed it to a staff to rush it to the Sick and Newborn Care Unit (SNCU).

\section{Towards truly respectful maternity care}

We highlight Deepa's case because it shows how complicated and tense the labor rooms can become. The staff did try to help Deepa and her baby by taking the blood pressure at the time of her admission and checking on the fetus's heartrate several times during labor. Yet, they behaved in ways that made the child-birth experience for Deepa disrespectful and abusive.

While witnessing disrespect and violence that pregnant women went through in delivery rooms, and while talking to the staff, a question that never left our minds was: Why are these women subjected to such behavior? And, what triggers such behavior of the staff? The answer to these questions has at least three parts: one, staff's burn-out from too many patients and long shifts; 
two, the lack of knowledge among staff on how to deal with the legitimate stress of a life and death situation; and three, a highly unequal society where it is socially acceptable to victimize lowranking people.

In an informal discussion, a doctor shared with us that sometimes doctors and nurses worry for their patients and they have trouble controlling their emotions and fears, so they try to motivate the patient with violence. "[During my residency years], I have been in that situation", an assistant professor in a Medical College, also a gynecologist, told us with a smile. The professor added, "students [in medical institutes] are overworked, and you can clearly see that frustration on their face. They have long shifts, often, as long as 16 hours in a row... There is so much that they are expected to do during the shift -see patients in OPDs, attend deliveries, work in the Emergency Room, sit outside wards, and then on top of all this fill out the paperwork for each of these. So, doctors prioritize. Amid all the chaos, there is just one thing they end up focusing - is the patient alive?"

The professor was correct in saying that the resident doctors and staff nurses are overworked. In a 135-beded tertiary care facility, there were only about a dozen residents to look after patients. In several instances, we saw staff nurses multitasking while attending multiple women who were in their second stage of labor. But the burn-out human resource theory, at best, can only partially explain the labor room violence that we witnessed.

The second reason for extensive labour room violence seems to be related to systemic issues such as the inflexible way in which maternal and infant care is set up and the lack of training among staff. The Labor Room Quality Improvement Initiative (LaQshya) ${ }^{\text {ii }}$ guideline, issued by the Government of India, suggests staff to 'not insist [a woman in labor, to lie] in the conventional lithotomy position' (Ministry of Health and Family Welfare 2017: 2). However, in Deepa's delivery the staff had her lie down on her back for the whole time. It is possible that if Deepa had been allowed to push in the way that felt more comfortable to her, and in which gravity helped her baby descend, she may not have gotten so tired, and the baby's heartbeat might not have dropped. But labor rooms in India are not set up to allow women to push in other positions, and staff is not trained to conduct deliveries in different ways. As a result, the staff quickly resorted to aggressive methods like applying fundal pressure on a woman's abdomen. And when fundal did not work, and the staff had anticipated fetal distress, their first reaction was frustration and aggression. 
In Deepa's case, the attending staff was ill-trained in dealing with the legitimate stress of a life-or-death situation. But at the same time, there also seems to be an overarching and inherent culture of abuse in healthcare settings wherein abusing pregnant women crying out of pain is considered a norm. As documented in an undercover report from a maternity hospital, doctors seem to informally learn this culture of abuse while in training (Chattopadhyay, 2015). Many medical students believed that slapping a patient was a "rite of passage" and thus something to celebrate. Given that doctors hold significant power in India's hierarchical healthcare settings, it is likely that their approval and exercise of violence gives sanction to other staff members and thus initiates a culture of abuse in the healthcare settings.

Relatedly, another driver for violence in labor rooms is the prejudices that the staff holds against the patients. A majority of doctors and nurses in India come from socioeconomically advantaged backgrounds, whereas most maternity patients served in the public hospitals are from poor and socially disadvantaged backgrounds. This inequality between the service providers and patients, in conjunction with a highly unequal setting, results in normalized violence and abuse.

\section{Policy changes as a way forward}

Given this reality, urgent steps must be taken to ensure truly respectful maternity care in government health facilities. While we work towards addressing the structural issue of social inequality, medical education and healthcare staff training institutions should include curricula on ethical patient-practitioner relationships and justice-oriented healthcare provisions. Staff should be oriented in patient-centered care that can not only improve health outcomes but also have positive impacts on staff's morale and productivity. Training birth attendants on how to manage stress and different positions of pushing during labour process should become a priority. The target-driven approach to family planning in India must end and attempts to diversify contraceptive choices and improve consent and counseling should be ensured. And finally, to prepare pregnant woman mentally, health care providers should talk to them and their family members beforehand on what the delivery may involve and make them aware of the possible issues that may come up.

Bureaucrats and policymakers, just like any other service provider, should devise ways to measure the quality of services that citizens receive. One can learn the most by talking to the patients, who in this case incorrectly are called "beneficiaries". Governments should set up systems to get patient's feedback on the quality of care they received. Doing all this is likely to be a 
steppingstone for improving culture in health institutions more broadly. And given that we know that public hospital deliveries have significant child health benefits over private facility and home births, it will also help thousands of babies by attracting pregnant women, who seek delivery care, to public hospitals. Improving patient experience and making maternity care respectful in government hospitals is a direct path to enhancing health and human development in India.

\section{References}

Chattopadhyay. S. (2015). 'I can't take it anymore': Sights and awful sounds from the labour room of an Indian public hospital. Scroll.in.

Coffey, D. Srivastav, N., Priya, A., Vermad, A., Spears, D. (2020). Excess neonatal mortality among private facility births in north India. [Preprint].

Kilpatrick, S., \& Garrison, E. (2012). Obstetrics: normal and problem pregnancies. Obstetrics: normal and problem pregnancies. 6th ed. Philadelphia, PA: Elsevier, Inc, 267-288.

Ministry of Health and Family Welfare. (2017). LaQshya- Labour Room Quality Improvement Initiative Guideline. Available from http://nhsrcindia.org/sites/default/files/LaQshya\%20Labour\%20Room\%20Quality\%20Improvement\%20Initiative\%20Guideline.pdf. ） (last accessed 17 January 2021).

Sharma. K. 2016. Two years after 18 women died at tubectomy camp, little has changed at India's sterilization drives. Scroll.in.

\footnotetext{
${ }^{\mathrm{i}}$ The lithotomy position is one in which a woman in labor lies on her back with her legs up, supported by straps or stirrups. Although nurses and doctors often find the lithotomy or supine (on one's back) positions convenient when attending births, research suggests that pushing in the upright position is more effective, especially for first time mothers (Kilpatrick \& Garrison, 2012).

ii In 2017, the Ministry of Health and Family Welfare, India, launched the Labour Room Quality Improvement Initiative (LaQshya). One of the main stated aims of the LaQshya guideline is to enhance the satisfaction of beneficiaries by ensuring 'Respectful Maternity Care' (RMC) at public health facilities.
} 